\title{
Discussion on the Modeling Language in Decorative Painting Teaching
}

\author{
Qunying $\mathrm{Li}^{1, \mathrm{a}}$ \\ ${ }^{1}$ Department of Art Design, Shan Dong Vocational College of Light Industry, Zibo 255300, China. \\ aliqunying2000@126.com
}

Keywords: Decorative Painting Teaching, modeling Language, concrete Form, deformation

\begin{abstract}
The modeling language training in decorative painting teaching is manifested as concrete form and abstract form. The concrete modeling language puts extra emphasis on showing generalization exaggeration, displacement reconstruction, shape modeling, planarization of shapes and other contents. The abstract modeling consists of accidental modeling and constitutive abstract modeling. Decorative modeling appears to be vigorous, or tenuous, or clumsy, or untrammeled in language. It is an ideal world with diversified characters; while its various forms and profound implications are a kind of unique artistic beauty. By using the laws and rules of formal beauty, decorative paintings emphasize the autonomy of subjective creation in the aesthetic aspect, and stress the diversified awareness and creation of beauty.
\end{abstract}

\section{Introduction}

"Decorative painting is a very broad field. Those paintings pertaining to or decorating the environment, those paintings pertaining to or decorating objects, those paintings pertaining to production process as well as those decorative paintings paying attention to forms all belong to the field of decorative paintings[1-4]. They are all decorative paintings or belong to paintings of decorative style." By using the laws and rules of formal beauty, decorative paintings emphasize the autonomy of subjective creation in the aesthetic aspect, and stress the diversified awareness and creation of beauty[5-7].

The concrete form refers to the objects objectively existing in the nature, including such natural forms as people, animals, mountains and rivers, as well as such artificial forms as buildings, household utensils, and means of transport. Deformation means changing the original appearance of natural forms and re-creating the natural images. The constitution principle - "the images in nature can be reduced to simplified spheres, cones and cylinders" put forward by Cezanne simplifies the natural images into geometric shapes, and conducts artistic processing for the trivial, scattered and non-typical characteristics of natural images in an inductive and general artistic language in order to make them neat, orderly and regular[8-10].

\section{Generalization exaggeration}

Decorative paintings emphasize the unity of emotions and expressions, aiming to purify and process the aesthetic experience from life to form patterned and orderly typical decorative images. It comprehensively reflects the high generalization of artists' aesthetic awareness and the ability of mastering and grasping the essence of art, changing the usual proportion of natural images from the visual aesthetics of pictures. For shape handling, it can make round objects rounder, square objects squarer, obese figures more obese. In the exercise of making exaggerated changes in the main characteristics of images, it's also required to appropriately adjust their local shapes so as to maintain the overall relationship coordination of paintings. The inductive strengthening of natural images should be combined with the component factors of paintings. Conciseness does not mean simplicity, otherwise it will look plain. Appropriate exaggerated changes can enrich the paintings, but exaggeration should be based on induction. Only when you are good at discovering the beauty of life can your artworks be unconventional and fresh. 


\section{Displacement reconstruction}

Decorative painting contains diversified reverse thinking associations. In order to strengthen one's individual will, we may essentially destroy and decompose natural shapes, i.e, displacing the natural form of images, breaking them down into several free shapes, and then recombining them to form a novel and extraordinary picture effect. In the same picture consisting of different combinations, through the borrowing and conformal concept of shapes and forms, the duplication and overlap of similar shapes, and the application of Montage Technique, the interspersed combination between shapes and images is given full play, and the imagination of art is fully developed.

\section{Shape modeling}

The composition of Chinese traditional decorative patterns - "two-dimension series, suitable decorative patterns" is the typical painting of shape modeling as well as the basic constitution form of decorative arts. It requires being greatly restricted by environment, border, shape, size, area and so on within a certain scope, designing and making layout in limited space. In the designing process, the principle of "taking advantages with shapes" should be taken into account. Besides, it also requires having certain adaptability to changes as well as organization and coordination ability. When we induce and decompose the natural forms, we should give full play to the artistic imagination. Art comes from life, but is different from life. Imagination is the life of art creation. Our living environment, experiences, customs, folklore, weddings \& funerals, marriage as well as other themes and fragments, are favorable conditions for our imagination and deformation, i.e., conducting exaggerated deformation for natural images, idealizing the shapes that cannot be reached in real life and vividly expressing them. For example, in the character modeling of Dunhuang Frescoes - "Flying Apsaras", the elegant figures with streamers flying in the air are playing melodious music, just like the old saying, "With flying clothes, all frescoes appear to be vivid", which fully expresses the romantic mood of ancient people.

\section{Planarization of shapes}

The line modeling in the line-drawing technique of meticulous style paintings in deep colors is not only an important means of decorative painting training, but also the concrete manifestation of planarization. "Lines" not only are used to outline the images, but also require showing the unique features of "lines". Lines can express length, direction, strength, position, thickness and speed, and have an independent feature. When outlining a contour, it emphasizes the big transition of the contour and the front and rear positions of shapes. "It is required to emphasize the unity relation of lines and shapes when handling shapes and lines." Conduct induction and unification for the basic shape that constitutes general characteristics and use the volume of geometry to generalize the overall relationship. For example, the human body structure lays emphasis on the connection of big movement lines in respect of the basic shape constituting individuality. At the same time, it also pays attention to the smoothness and strength of lines and the harmonious rhythmic beauty of the whole painting.

Object shape modeling "highlights the outer contour features of image, and draws the people's attention to the shape edge. In the expression of shapes, the stone portrait of the Han Dynasty is a typical example. It abandons tedious details, and generalizes and exaggerates the expressive dynamic state of figures, thereby, enhancing the overall unity relation between decoration and the overall painting.

It consists of the changes of such modeling elements as points, lines, surfaces and shapes, without direct implication. Through the combination and transfer of some abstract information, it enriches people's imagination. Absolutely abstract does not exist and the abstract form corresponds to the concrete form. On the one hand, abstract modeling comes from the spiritual feeling generated due to the objective external stimuli, and then inspires creativity. On the other hand, it means the rational 
and orderly combination \& design of the basic elements constituting modeling, including points, lines, surfaces and shapes, thereby, forming a constitutive abstract form.

\section{Occasional Abstract}

The common object forms we see in the nature can stimulate the inspiration in the twinkling of an eye, triggering the new symbolic language such as "spiral lines on the water surface skipped by the raindrops, and the micro-morphology under the microscope..." According to Kandinsky, "An abstract painter accepts all his incentives, not from any piece of the nature, but the nature as a whole and its various manifestations. The works are created and accumulated from his inner heart." The new artistic image sources come from occasional subconscious revealed forms, the stimulating association with the existing figures and patterns, as well as the dreamlike imaginary modeling symbols.

\section{Constitutive Abstract}

The basic elements of modeling can be used as the decorative drawing methods which include dot, line, plane, form and color, emphasizing sense of rhythm and its beauty of the combined elements in the picture. Here rhythm means the harmony and unification of dynamic movements and artistic images of the overall images in the picture. The outline undulation of the same image, density arrangement of dots, the unrestrained rigidness and softness of lines, immediate repetition of the similar figures, inclined dynamic of the movement lines, static and movement of colors and texture structure of pictures all determine the dynamic-static comparison between images and permutation combination. The dynamic rhythm of structure is directly reflected on the dynamic movements, image intensity and configuration rhythms. We feel artists' sublimation of rich emotions through the interaction among dots, lines, planes and forms.

\section{Summary}

The modeling training of decorative paintings is a continuously accumulative and creative process, which can make progress with the continuous improvement of people's artistic accomplishments and aesthetic consciousness. Decorative modeling appears to be vigorous, or tenuous, or clumsy, or untrammeled in language. It is an ideal world with diversified characters; while its various forms and profound implications are a kind of unique artistic beauty.

\section{Acknowledgements}

The authors would like thank the 2015 Annual Vocational Education Reform Projects of Shandong Province (No. 2015040) for financial support.

\section{References}

[1] J. L. Chen, Decoration Painting, second ed., The Central South University Press, Changsha, 2008.

[2] X. G. Zhang, Z. S. Long, Decoration Painting, first ed., Nanjing University press, Nanjing, 2010.

[3] S. Y. Liu, Creative and Performance of Decorative Pattern, second ed., China Textile \& Apparel Press, Beijing, 2008.

[4] Yimin, Zhang. Decorative Painting, first ed., Shangdong Art Publishing House, Jinan, 1997.

[5] L. Q. Zhou, Decoration Painting, second ed., Hunan University press, Changsha, 2005.

[6] Y. Gao, Q. F. Zeng, Decorative Painting, first ed., Harbin Engineering University press, Harbin, 2011. 
[7] J. Y. Lin, Design color, third ed., Beijing, Higher Education Press, Beijing, 2014.

[8] Q. Zhu, Design color, first ed., Huazhong University of Science and Technology press, Wuhan, 2011.

[9] P. X. Wu, Decoration Painting, first ed., Southwestern Normal University Press, Chongqing, 2006.

[10] Y. Gao, X. Jiang, D. Wei, first ed., Ordnance Industry Press, Beijing, 2014. 